\title{
ESTRATEGIAS PARA CONSTRUIR LA PAZ EN LA REGIÓN: CONTRIBUCIONES DE LA FACULTAD DE EDUCACIÓN DE LA UNIVERSIDAD DE NARIÑO
}

\section{STRATEGIES TO BUILD PEACE} UNIVERSITY OF NARIÑO

\section{Nelson Torres Vega ${ }^{1}$}

\section{Francisco Javier Torres Martínez ${ }^{2^{* *}}$}

\section{Edgar Guillermo Mesa Manosalva ${ }^{3^{\text {t+x }}}$}

Universidad de Nariño Grupo de investigación

para el desarrollo de la educación y la pedagogía - GIDEP

\footnotetext{
$1 \quad$ Doctor en Educación de la Universidad de Nariño. Magíster en Modelos de Enseñanza Problémicade la Fundación Universidad Incca. Magíster en Educación, Orientación Profesional y Consejería Escolar de la Universidad Pedagógica y Tecnológica de Colombia. Licenciado en Psicología Educativa y Administraciónde la Universidad Pedagógica y Tecnológica de Colombia. Decano Facultad de Educación y miembro Grupo GIDEP de la Universidad de Nariño. neltorres58@hotmail.com 2 "Doctor en Ciencias de la Educación de la Universidad de Nariño. Magíster en Mercadeo Agroindustrial de la Universidad Jorge Tadeo Lozano. Especialista en Proyectos de desarrollo del convenio Universidad de Nariño y Escuela Superior de Administradores Públicos. Especialista en Docencia Universitaria de la Universidad de Nariño. Especialista en manejo postcosecha en frutas y hortalizasdel Convenio Sena Reino Unido. Ingeniero Agronómico de la Universidad de Nariño. Miembro Grupo GIDEP de la Universidad de Nariño.franjatm@hotmail.com

3 Doctorandode la Universidad de Granada. Magíster en Modelos De Enseñanza Problemica de la Fundación Universidad Incca De Colombia. Especialista en Estudios Latinoamericanos de la Universidad de Nariño. Licenciado en Educación y Ciencias Religiosas de la Universidad Pontificia Bolivariana. Licenciado en Filosofia de la Universidad Santo Tomas. Profesor y miembro Grupo GIDEP de la Universidad de Nariño.Manosalva50@hotmail.com
} 


\section{RESUMEN}

El presente artículoes derivado del proyecto de investigación: Estrategias pedagógicas para construir la paz en la región: Contribuciones de la Facultad de Educación, Universidad de Nariño, auspiciado por la Vicerrectoría de Investigaciones, Posgrados y Relaciones Internacionales - VIPRI y desarrollado por el Grupo de Investigación para el Desarrollo de la Educación y la Pedagogía - GIDEP, Línea de Investigación "Pedagogía Social". Tiene como propósito analizar las estrategias pedagógicas para construir la paz en la región, como una contribución de la Facultad de Educación. En el marco de los diálogos de paz se ha invitado a todas las universidades del país a generar sugerencias, a presentar propuestas, iniciativas y estrategias orientadas a construir una paz duradera que aporte al desarrollo económico, político, social y cultural.

Para tal efecto, se acudió a un proceso investigativo centrado en los docentes y estudiantes de los programas de la Facultad de Educación, caracterizado por los siguientes aspectos: la investigación se inscribe dentro del paradigma Cualitativo, con enfoque etnográfica y método de acción participativa,incluyendo interacciones entre los investigadores y los investigados.

Entre otras conclusiones se destaca que la Facultad de Educación de la Universidad de Nariño, en su Proyecto Educativo, concibe el concepto de la didáctica, como las teorías y prácticas que en coherencia con la fundamentación pedagógica y en consideración a la naturaleza, la historia y la epistemología de los saberes, permiten desarrollar procesos de transposición didáctica que conllevan a la apropiación consciente y reflexiva del conocimiento científico. Lo anterior, constituyen insumos insustituibles para la construcción de estrategias de paz con miras a fortalecer los procesos derivados del acuerdo de la Habana y el post-acuerdo, la cultura y la educación para paz, el pacifismo, la resolución de conflictos, la mediación, entre otros, para contribuir a la construcción de la paz en el departamento de Nariño.

PALABRAS CLAVE: Paz, conflicto, pedagogía, acuerdos de paz, estrategias.

\section{ABSTRACT}

KEYWORDS: Peace, conflict, pedagogy, peace agreements, strategies.

\section{INTRODUCCIÓN}

Este artículo derivado de la investigación referida, aborda el análisis de estrategias pedagógicas de los docentes de la Facultad de Educación para articularlas a múltiples interacciones con otros temas como la interculturalidad, la multiculturalidad, la cultura y educación de paz, el pacifismo, la resolución de conflictos, la mediación, entre otros, para contribuir a la construcción de paz en la región sur y fronteriza.

Colombia ha vivido toda clase de conflictos y violencias durante los últimos cincuenta años $y$ en los cinco gobiernos anteriores se han buscado alternativas de solución al centenar de conflictos que se han generado en todos los sectores sociales y políticos y no se han dado las condiciones, ni los recursos, ni los frutos esperados.

El departamento de Nariño, ubicado en la región suroccidental del país, se caracteriza por su diversidad étnica, cultural e histórica. Grupos prehispánicos habitaron sus territorios y en el transcurso de la conquista y colonización española se mezclaron dando origen a un mestizaje cultural, étnico, religioso, gastronómico, artesanal, idiomático y social. Persisten varios grupos entre los cuales se encuentran los pastos, los quillacingas, los awá, los ingas, los emberá, entre otros. 
Hoy el pueblo clama, quiere y busca la paz, y el actual gobierno tiene la voluntad para generar procesos en la construcción de la misma. En este anhelo se inscribe esta investigación bajo el planteamiento de si es posible construir la paz en el departamento de Nariño, con los aportes teóricos y metodológicos de los docentes de la Facultad de Educación de la Universidad de Nariño. Los aportes son conocimientos, principios, prácticas y experiencias sociales, es decir son recursos intelectuales, sociales, materiales- técnicos y pedagógicos para construir la paz en Nariño.

La paz es en primer término un asunto de educación, es decir, en las aulas se aprende y se generan las estrategias que propician la convivencia, la solidaridad, el diálogo entre los sujetos que intervienen en el acto educativo. Por esto es importante que desde la educación se generen escenarios óptimos y adecuados para la consolidación de la cultura de paz. La paz es tema de estudio sistemático, interdisciplinar y transdisciplinar y está presente en investigaciones de alto impacto en universidades europeas, norteamericanas, latinoamericanas y colombianas. La Facultad de Educación de la Universidad de Nariño asume el compromiso de trabajar por la Paz y a través de acciones significativas que aportan permanentemente a la construcción de escenarios que propician ambientes de reconciliación en la región. La pregunta central es la siguiente: ¿Qué estrategias pedagógicas de los docentes de la Facultad de Educación contribuyen a la construcción de la paz en la región?

\section{LA PAZ Y LOS CONFLICTOS}

En la naturaleza todos los seres que la habitan poseen poder, energía y por esta razón se transforman. Los seres vivos en su dinámica cotidiana, en su acontecer diario necesitan e interactúan en el poder, porque siguiendo a Aristóteles, todo es acto y potencia. El género humano es poder en sí mismo, porque es energía, fuerza, pasión, razón, bondad, odio y en todo ello radica el poder.

El conflicto es connatural a la condición humana, es parte de su esencia, es lo que permite su crecimiento y su desarrollo. El conflicto es fuente de crisis, porque genera asombro, caos, desorden, desespero, ansiedad, impaciencia; es decir, desequilibrio. $Y$ cuando el ser humano se descompensa, entonces sufre y comienza la búsqueda para salir de esa situación.

El manejo adecuado del conflicto permite que los seres humanos se entiendan y comprendan; sin embargo, en la práctica se encuentran diferentes lenguajes, pensamientos, creencias religiosas, entre otras. Frente a lo anterior, siempre habrá divergencias, obstáculos, y en esa situación el conflicto aparece y la forma de comprenderlo e interpretarlo requiere de la actitud pacífica o violenta del ser humano. Lo anterior, lo corrobora Muñoz (2013), y ratifica que el conflicto es la relación entre los seres humanos, individuos, grupos y especies de una manera compleja, el cual se puede dirimir de manera pacífica o violenta.

Una forma de direccionar la resolución de los conflictos a través de la vía pacífica, es mediante el estudio de la paz y las mediaciones que son conceptos que nos permiten mirar con optimismo las relaciones interpersonales y provocan una actitud permanente de reflexión, análisis y actitud crítica frente a los acontecimientos.

Según Bruner (1987), la cultura es un conjunto de pensamientos (cosmovisiones), sentimientos (axiología) y prácticas sociales (comportamientos) compartidos por un grupo social; desde el nacimiento hasta la muerte somos sujetos culturales. Somos sujetos educables y por esto los principios de educabilidad y enseñabilidad se justifican en razón de su finalidad. 


\section{LA CULTURA DE PAZ}

Según la Organización de las Naciones Unidas para la Educación, la Ciencia y la Cultura -UNESCO (1998), la Cultura de Paz involucra valores, actitudes y comportamientos sin el uso de la violencia con el fin de prevenir los malos entendidos para alcanzar la paz entre las personas, los grupos y las naciones. El diálogo y la negociación, son mediaciones aprendidas, que pasan por el tamiz de la experiencia y conocimiento familiar y social; en rigor la cultura de paz es en esencia un asunto educativo, político y social.

La cultura de paz se promueve por medio de la educación, por lo tanto, los contenidos curriculares de los programas educativos deben asumir como su eje trasversal, los componentes formativos que tienen que ver con la construcción de paz, lo cual indica que las instituciones educativas son el escenario adecuado e idóneo para la consolidación de la cultura de paz.

De igual modo, la cultura de paz promueve la participación democrática, la comprensión, la tolerancia y la solidaridad; en consecuencia, se requiere propiciar la comunicación participativa y la libre circulación de información y conocimientos. La cultura de la paz es fundamental porque busca construir escenarios de convivencia y tolerancia en la región, en la ciudad y en la universidad.

Fisas (2011:3), conceptúa sobre la educar en la cultura de la paz:

La cultura de paz es una tarea educativa que pasa por educar en y para el conflicto, en desenmascarar la violencia cultural y el patriarcado, en educar para la disidencia, el inconformismo y el desarme, en responsabilizarnos, en movilizarnos, en transformar los conflictos, en llevar a cabo el desarme cultural, en promover una ética global y en buscar un consenso fundamental sobre convicciones humanas integradoras, entre otras.

En el quehacer docente, las aulas de clases son escenarios óptimos para la consolidación de la cultura de paz, porque son el espacio para la socialización de temas pertinentes y del interés de los estudiantes. En este sentido, Mayor (2003:17), afirma:

La educación para la paz ha de ser considerada como contenido transversal de la educación. La situación actual, los conflictos interétnicos e interculturales, la exclusión y la marginalidad, las sociedades pobres y las ricas son cuestiones que reclaman una educación para la paz.

\section{EDUCAR PARA LA PAZ}

La educación debe constituir una herramienta para la construcción de paz en los diferentes espacios interculturales, de ahí que es un compromiso de todos los actores involucrados en los escenarios educativos. En este contexto, la educación constituye un servicio público que tiene una función social, por esto es pertinente preguntar las acciones formativas desarrolladas por los educadores en los niveles de la educación. Al respecto la Constitución Política de Colombia, en el art. 67 dice:

La educación es un derecho de la persona y un servicio público que tiene una función social; con ella se busca el acceso al conocimiento, a la ciencia, a la técnica, y a los demás bienes y valores de la cultura.

La educación es un derecho, y los Derechos Humanos - DDHH son el contenido y estructura de la educación. ¿Qué sería de la educación sin derechos humanos? Educar en los derechos humanos, en el respeto, la tolerancia, el amor, la amistad, el diálogo es un imperativo 
social. La Declaración Universal de los DDHH (1948), considera que la condición humana está centrada en la dignidad, entendida como aquello que hace a los hombres merecedores del respeto y la estimación de los demás y de sí mismos.

\section{UNIVERSIDAD Y PAZ}

La paz es un asunto de profundas repercusiones sociales, políticas, económicas y educativas y son las instituciones educativas las primeras llamadas a contribuir para asumirla en sus currículos y estudiarla con rigor y seriedad. Pero será la Universidad la primera en proponer la paz como objeto de estudio interdisciplinar y transdisciplinar, crear y promover espacios para su debate, gestión y promoción.

Con relación a la contribución que se espera de la formación universitaria para alcanzar la paz, Bermúdez (2001; 221), anota lo siguiente:

(...) si bien existen áreas claves a fortalecer en nuestro conocimiento de manera que afecten el cotidiano como son (entre otras) las relativas al desarrollo individual y social, a la equidad, los derechos humanos, al derecho internacional humanitario, al tratamiento de los conflictos, a la ética, a la responsabilidad civil, a la pedagogía, al desarme simbólico que a veces es más grave que el material, o a los procesos cognoscitivos asociados a su contexto de origen para ser por lo menos más conscientes de los logocentrismos, se debe recalcar que desde cualquier campo del conocimiento se puede aportar a la paz.

En concordancia a lo anterior, la Universidad de Nariño tiene el compromiso de liderar, en la región, el estudio científico de la paz, proponer acciones para su construcción, dedicar recursos humanos y económicos para su promoción, avalar y valorar iniciativas encaminadas a la transformación pacífica de los conflictos, fomentar el diálogo y la cooperación interinstitucional para la búsqueda de soluciones a los problemas de la región. En este sentido, la Universidad de Nariño, está comprometida con la construcción con acciones de paz territoriales, las cuales se intuyen en el Plan de Desarrollo 2008 - 2020, Pensar la Universidad y la Región (2008), que anota lo siguiente:

La contribución de la Universidad a la construcción de la región soñada se hará a través de las funciones que le son propias. Los currículos transdisciplinares permitirán el acercamiento a la comprensión holística de la región y la auto comprensión de la Universidad como parte consustancial de aquella; al tiempo que permite la formación de profesionales capaces de pensar e imaginar mundos mejores y contribuir a su construcción(p. 26).

Abordar la cultura de la paz en ambientes educativos es posibilitar que el pensar, sentir y obrar de la comunidad académica a partir del aula de clase, en la biblioteca, laboratorio y otros escenarios universitarios. La cultura de paz ronda todos los ámbitos de la universidad y permea el quehacer educativo del diario vivir.

En este contexto, la cultura de paz está relacionada con procesos de enseñanza, aprendizaje mediante estrategias, técnicas, métodos, metodologías para realizar su función de enseñar, pero unida a la psicología cognitiva, al desarrollo socio-afectivo para cumplir con su función formadora y para lograr óptimos aprendizajes.

\section{FACULTAD DE EDUCACIÓN Y REGIÓN}

En la misión de proyecto educativo de la facultad se encuentra la orientación acerca de formar educadores de alta calidad ética, pedagógica 
y científica que les permita comprometerse en la transformación de las realidades y conflictos presentes en la región y el país. Una de las formas más expeditas para este propósito es la formulación de estrategias alternativas orientadas a construir la paz.

El estudio retoma los planteamientos de Freire y Giroux respecto a la pedagoga critica, cuya orientación central es la transformación de las realidades educativas a partir del compromiso político y éticos de los docentes. Por otra parte, se retoman los aportes del instituto de la paz y los conflictos de la Universidad de Granada, España respecto a la matriz que estudia los asuntos de la paz: paz y conflictos, noviolencia y resolución pacífica de los conflictos, género paz, mediaciones y empoderamiento pacifista. $Y$ en tercer lugar los conceptos provenientes de la UNESCO acerca de la Cultura de Paz.

\section{METODOLOGÍA}

El proceso investigativo se realizó con docentes de los programas de la Facultad de Educación de la Universidad de Nariño. La investigaciónse inscribe en el paradigma cualitativo, por cuanto las interacciones entre los investigadores y los investigados, evidenciaron experiencias, necesidades y significados de los sujetos participantes. Este enfoqueconceptualiza la realidad según el comportamiento, las actitudes y los valores de los sujetos estudiados (Martínez, 2006); y permite comprender las contribuciones educativas y pedagógicas de los docentes para la construcción de paz en la región, a partir de referentes pedagógicos, epistemológicos, antropológicos y sociológicos.

Esta investigación de carácter etnográficoeducativo,comprometió a los investigadores con los docentes en su formación académica, investigativa, prácticas sociales y educativas al servicio de la región. Considera la inquietud por investigar sobre la paz, los conflictos, la interculturalidad, la multiculturalidad, la complejidad, el poder, el pacifismo y la cultura; marca una ruta de indagación, es decir, la forma cómo se ha construido el objeto de análisis (Martínez, 2006).Se acudió al método de Investigación Acción Participativa -IAP, el cual permitió identificar las acciones que contribuyen a la construcción de paz en el post-acuerdo 4 .

La investigación incluyó las fases de: 1) Planeación, en la cual se recopiló información, ideas, conceptos y experiencias de los docentes sobre estrategias pedagógicas, paz y conflictos; se analizó esta información mediante la identificación de categorías para luego transformarlas en conceptos más amplios; 2) Reflexión, permitió establecer su efecto sobre la problemática estudiada y se realizaron los ajustes correspondientes; y 3) Evaluación,incluyódatos generados en los procesos de reflexión, la percepción de los participantes, con relación a los resultados obtenidos y el análisis de los indicadores establecidos en la fase de planeación.

Los instrumentos utilizados para la recolección de información primaria fueron, las entrevistasindividuales y lostalleres focales ${ }^{5}$, seleccionados bajo el criterio de intencionalidad y representatividad del grupo (Martínez, 2006); la observación participante que permitió interactuar con el grupo de docentes, en forma consciente, crítica y flexible.

Para complementar la información primaria, se recurrió a: revisión bibliográfica de archivos institucionales, libros, revistas, trabajos de grado, relacionados con referentes pedagógicos,

4 Post-acuerdo: entendido como los escenarios políticos, económicos, sociales y culturales, derivados del Acuerdo de Paz firmado el 24 de noviembre del 2016, entre el Gobierno del presidente de Juan Manuel Santos y las Fuerzas Armadas Revolucionarias de Colombia - Ejército del Pueblo (FARC-EP).

5 La técnica de los grupos focales (Bonilla 1997,102), "se ha convertido en uno de los principales instrumentos de los métodos de indagación rápida, desarrollados para obtener información ágil que posibilite dar respuesta en el corto plazo a las urgentes necesidades sociales que se investigan y, al mismo tiempo, responda de manera rigurosa a las exigencias del método científico". 
etnicidad, cultura, paz, violencia, conflicto y cultura de paz.

El procesamiento de la información incluyó un análisis descriptivo, que implicó seleccionar, clasificar y valorar las fuentes primarias; luego, se establecieron categorías y subcategorías para comprender los significados que los docentes asignan a los referentes pedagógicos para construir la paz en el post-acuerdo. Posteriormente, se interpretó la información para producir un referente teórico coherente al objetivo central de la investigación, que inspiró la generación de escenarios para la construcción de la paz.

\section{RESULTADOS Y DISCUSIÓN}

Los resultados derivados del análisis de la información primaria y secundaria, evidencia la existencia de estrategias pedagógicas que contribuyen a la construcción de paz en la región desde el contexto académico de los programas de la Facultad de Educación de la Universidad de Nariño.

\section{CARACTERIZACIÓN DE LA PRÁCTICA PEDAGÓGICA DE LOS DOCENTES}

Con relación al desarrollo de la práctica pedagógica ${ }^{6}$ de los docentesen su dinámica curricular, se identificaron las categorías relacionadas en la tabla $\mathrm{N}^{\circ} 1$

Estas categorías están en relación directa con el quehacer cotidiano del docente en el proceso de enseñanza aprendizaje de los contenidos temáticos de las asignaturas de los programas

\footnotetext{
6 Práctica pedagógica: "se define como la construcción teórico-formal, que representa la realidad estudiada y se fundamenta científicamente en las teorías de la educación y la pedagogía; metodológicamente se basa en los diferentes enfoques de investigación y responde a una necesidad histórica concreta: la formación del maestro crítico, creativo, reflexivo, investigador y comprometido con los valores de la convivencia, el pluralismo, la solidaridad y la justicia social". Universidad de Nariño. (2014). Reglamento Practica pedagógica integral e investigativa-PPII, Facultad de Educación. p. 10.
}

de licenciatura objeto de estudio.

En este contexto se evidencian estrategias para la construcción de paz; por ejemplo, el diálogoque se deriva de la dinámica curricular en el aula de clase, es un insumo sustantivo para lograr acuerdos entre los sujetos para dirimir conflictos, desacuerdos, diferencias, tensiones y similares. En la medida en que estas circunstancias se den en el aula se crea un clima de armonía que contribuye a la construcción del conocimiento y la paz, esto se replicará en la práctica pedagógica de los docentes en formación y finalmente va a llegar a la sociedad. Lo anterior se ratifica en la respuesta de un docente encuestado, así:

(...) el diálogo permanente con el estudiante y la realidad del contexto, yo como docente dialogo con mi familia, con el taxista, con el de la moto, con la del mercado, con un amigo o desconocido de la calle. Concluyendo, puedo precisar que desarrollo mi práctica pedagógica orientado al lineamiento institucional Universitario - Universidad de Nariño, que da la facultad de educación y que determina cada programa; aplico teoría social, cultural y epistemológica en contexto de mis estudiantes de Lengua Castellana y Literatura, Ciencias Naturales, Inglés y Francés, Lengua Castellana e Inglés, en diálogo - conversatorio y crítica social ${ }^{7}$.

\section{INFLUENCIA DEL ENTORNO EN LA PRÁCTICA PEDAGÓGICA DE LOS DOCENTES}

Se indagó sobre la influenciadel medio geográfico, cultural, económico, ambiental, social y político en la práctica pedagógica de los docentes. Se identificaron las categorías relacionadas en la tabla $\mathrm{N}^{\circ} 1$.

Se reconoce que las prácticas pedagógicas de los docentes están determinadas por

$7 \quad$ Respuestas de los docentes participantes en la aplicación de la encuesta. Noviembre 22 del 2017. 
los contextos particulares en los cuales se ejerce esta actividad. Dichos contextos se caracterizan por sus dinámicas permanentes entre los actores de los diferentes colectivos comunitarios y de éstos con el ámbito educativo (interacciones entre miembros de la comunidad educativa y organismos comunitarios en contextos particulares). Esta percepción, la explica un docente encuestado en la presente investigación:

El contexto en el cual se desarrolla la práctica pedagógica, determina mi ejercicio profesional, ya que es allí en donde se ponen a prueba todos los procesos académicos, de docencia,investigación e interacción social que desde la universidad se proponen ${ }^{8}$.

\section{CONCEPTO DE PRÁCTICAS SOCIALES}

Con respecto al concepto sobre Prácticas Sociales, los docentes se refierena "las interacciones de los maestros en formación y los licenciados como tal en las instituciones educativas en relación con toda la comunidad educativa: docentes, estudiantes, padres de familia, personal administrativo, servicios generales, y comunidad en general"9; otra percepción refiere a "las acciones concretas aplicadas desde las áreas - ciencias a la realidad humana vivida, es confrontar teoría y prácticas, para transformar la realidad a través del cambio."10, además, como "el ejercicio contextualizado del ser humano en un entorno social se constituye en una práctica social de tal manera que todo ser humano en el ejercicio propio de su condición desarrolla prácticas sociales"11.

Los conceptos se complementan e incluyen la totalidad de los actores que interactúan en los procesos sociales, en el proceso de enseñanza-

Ibíd.
Ibíd.
Ibíd.
Ibíd.

aprendizaje y de este hacia la familia, la sociedad y el entorno cultural, político y social. En los conceptos emitidos por los docentes, se identificaron las categorías relacionadas en la tabla $\mathrm{N}^{\circ} 1$

\section{CONCEPTO DE PEDAGOGÍA SOCIAL}

Para los docentes la Pedagogía Social es considerada como "el impacto que se genera con la práctica pedagógica en los procesos de formación y educación en las instituciones educativas, generando una verdadera transformación social, para un buen ser"12; de igual manera"la sociedad es la fuente y el sentido del ser de la pedagogía. Toda la Pedagogía es Social, porque el ser de las disciplinas no es reproducir solo la ciencia, sino, aplicar y además crear ciencia para la transformación y el cambio social"13. De la misma forma, se concibe:

(...) fundamentalmente en el acto educativo (todo escenario en donde el ser humano consolide su proceso de aprendizaje no solo en la escuela), es mediante la pedagogía social que se encuentran fundamentos: filosofías, epistemologías; disciplinarias y didácticas que son argumentados desde esta arca fundamental en la sociedad. ${ }^{14}$

En las respuestas de los docentes, se identificaron las categorías relacionadas en la tabla $N^{\circ} 1$

\section{ESTRATEGIAS PARA LA CONSTRUCCIÓN DE PAZ EN LA REGIÓN}

Una vez analizada la información primaria de esta investigación, se identificaron las siguientes estrategias para la construcción de paz en la región:

Diálogo: es la conexión del docente con el estudiante mediante los conversatorios y la crítica social en la dinámica curricular al interior del aula de clase. Como ya se hizo referencia

\begin{tabular}{ll}
\hline 12 & Ibíd. \\
13 & Ibíd. \\
14 & Ibíd.
\end{tabular}


en el aparte correspondiente, está estrategia para alcanzar la paz, es un insumo sustantivo para lograr acuerdos entre los sujetos para dirimir conflictos, diferencias y tensiones, circunstancias propias del aula de clase creando ambientes de armonía para la construcción del conocimiento y la paz.

Acompañamiento: es una asesoría y apoyo permanente que constituye una mediación pedagógica, por parte de los docentes en la dinámica curricular del proceso de enseñanzaaprendizaje. Como estrategia, el maestro se constituye en un guía y orientador de todos los procesos formativos en el anhelo de conseguir la paz desde el aula hasta la sociedad.

Seguimiento y evaluación: consiste en la observación y análisis de fenómenos sociales, mediante procesos de reflexión del pensamiento crítico basado en la fundamentación teórica; además, acciones y comportamientos al interior del aula de clase para asumir correctivos y valorar los aprendizajes de los estudiantes. Como estrategia se refiere al hecho de reconocer resultados parciales y su respectiva retroalimentación, lo cual aporta elementos sustanciales para construir la paz desde el aula.

Sensibilidad contextualizada: hace referencia a potencializar a través de los sentidos el contexto del aula, la practica pedagógica y la interacción social en la comunidad educativa; entre otros elementos sustantivos, la reflexión se convierte en un componentetransversal de las estrategias utilizadas por los docentes en sus dinámicas curriculares para consolidar la paz en la región. Se trata de una estrategia ubicada en el plano subjetivo que permite reconocer las problemáticas, las sensibilidades, actitudes, sentimientos, disposiciones en el ámbito escolar como punto de partida para consolidar la paz desde el aula hacia otros contextos.

Encuentro de saberes: entendido como la transformación de las realidades a partir de la construcción de nuevos saberes, consecuencia de las interacciones entre los sujetos que habitan la escuela y sus conocimientos, roles, vivencias, expectativas y prácticas sociales. Está estrategia implica, entre otras,la noción de "otredad", que consiste en reconocer al Otro como individuo diferente y se asume su identidad; además, la interdisciplinaridad en relación con la construcción del conocimiento desde diversas miradas en el aula para consolidar la paz.

Identificando problemas: el docente es el llamado a reconocer la escuela como escenario de investigación que permita identificar problemas sociales, económicos, culturales, educativos y pedagógicos, para plantear accionesencaminadas al cambio social. Una estrategia para este fin, consiste en potenciar la sensibilidad del docente en su ámbito curricular, lo cual implica su preparación y aplicación de enfoques teóricos y metodológicos sobre la investigación in situ.

Transformación social: se refiere a los cambios sustanciales en la sociedad, a los cuales, se refiere Espinosa (2018), así:

Transformar una sociedad es una tarea que lleva tiempo, especialmente si esa sociedad viene de vivir episodios de retraso económico, de seguridad $\mathrm{y}$, sobre todo, morales.No es tarea fácil recomponer lo que llaman el "tejido social", principalmente cuando se han vivido periodos de violencia, crisis económica y ruptura de la infraestructura que le daba cohesión a la comunidad. Es algo que no sólo se logra con inversión financiera, también hay que invertirle al liderazgo y al "bien estar", con todo lo que la palabra implica.

En consecuencia de lo anterior, los cambios se miden por el impacto que estos producen en el tejido social, es decir, lograr el mejoramientoen el nivel de vida de los colectivos sociales y a la vez generar estrategias de construcción de paz. Este debe ser un compromiso permanente del docente, dentro y fuera de la escuela. 
Tabla 1. Síntesis de interrogantes, categorías de análisis y estrategias para construcción de paz

\begin{tabular}{|c|c|c|}
\hline Interrogantes & Categorías de análisis & $\begin{array}{c}\text { Estrategias para } \\
\text { construcción de paz }\end{array}$ \\
\hline $\begin{array}{c}\text { Caracterización de la } \\
\text { práctica pedagógica de los } \\
\text { docentes }\end{array}$ & $\begin{array}{ll}\text { - } & \text { Conversatorio y crítica social } \\
\text { - } & \text { Acto social } \\
\text { - } & \text { Cultura, mediada por la } \\
& \text { pedagogía } \\
\text { - } & \text { Reflexión del pensamiento crítico } \\
\text { - } & \text { Fundamentación teórica } \\
\text { - } & \text { Características de un maestro y } \\
& \text { su papel en la sociedad } \\
\end{array}$ & $\begin{array}{ll}\cdot & \text { Diálogo } \\
\cdot & \text { Acompañamiento } \\
\cdot & \text { Seguimiento y } \\
\text { evaluación }\end{array}$ \\
\hline $\begin{array}{c}\text { Influencia del entorno en la } \\
\text { práctica pedagógica de los } \\
\text { docentes }\end{array}$ & $\begin{array}{ll}\cdot & \text { Reflexión } \\
\cdot & \text { Práctica pedagógica } \\
\cdot & \text { Interacción social } \\
\text {. } & \text { Contexto }\end{array}$ & $\begin{array}{ll}\text { - } & \text { Sensibilidad } \\
& \text { Contextualizada }\end{array}$ \\
\hline $\begin{array}{c}\text { Concepto de Prácticas } \\
\text { Sociales }\end{array}$ & $\begin{array}{ll}\cdot & \text { Humana } \\
\cdot & \text { Realidad } \\
\cdot & \text { Transformar la realidad } \\
\text {. } & \text { Interacción social } \\
\text {. } & \text { Prácticas Sociales } \\
\end{array}$ & Encuentro de saberes \\
\hline $\begin{array}{c}\text { Concepto de Pedagogía } \\
\text { Social }\end{array}$ & $\begin{array}{ll}\cdot & \text { Impacto } \\
\cdot & \text { Social } \\
\cdot & \text { Interacción social }{ }^{1}\end{array}$ & $\begin{array}{ll}\cdot & \text { Identificando } \\
\text { problemas } \\
\text {. } & \text { Transformación social }\end{array}$ \\
\hline
\end{tabular}

Fuente: esta investigación

\section{CONCLUSIONES}

- Con respecto a la caracterización de la práctica pedagógica por parte de los docentes, es evidente que las vivencias expresadas por los sujetos participantes de los procesos de formación, están en función del diálogo, el acompañamiento y la evaluación que se han resignificado como estrategias para la construcción de la paz. Dichas estrategias se encuentran inmersas en la dinámica curricular escolar y son determinadas por los entornos geográfico, cultural, económico, ambiental, social y político en las prácticas pedagógicas de los docentes; no obstante, en ocasiones pasan desapercibidas en la cotidianidad del ámbito educativo.

- Con relación a la concepción de los docentes sobre las Prácticas Sociales, coinciden en que el encuentro de saberes constituye un eje central de las estrategias para la construcción de la paz en ámbito escolar. Lo anterior, como resultado de las múltiples interacciones entre los saberes que se generan a partir de la interdisciplinariedad 
y el reconocimiento al Otro como individuo diferente.

- Finalmente, los docentes aproximan la Pedagogía Social en torno a los cambios que se producen en el tejido social, es decir, lograr el mejoramiento en el nivel de vida de los colectivos sociales que rodean la escuela, y a la vez generan estrategias de construcción de paz.

- Es necesario promover una cultura de paz consistente en la formación sobre una serie de valores que determine las actitudes y los comportamientos de todos los sujetos que habitan la escuela. Lo encontrado en la investigación ratifica lo expresado por Mayor Zaragoza (2003:8), cuando afirma que la educación y la paz deben ser correlativas para superar los conflictos actuales de la sociedad y superar la exclusión, la marginalidad y los conflictos interétnicos e interculturales y superar la brecha entre las sociedad pobres y ricas

\section{REFERENCIAS BIBLIOGRÁFICAS}

Bermúdez Q, Suzy. (2001). La Universidad y la paz en Colombia. Nómadas (Col), núm. 14, abril. pp. 209-222. Universidad Central. Bogotá, Colombia

Bonilla-Castro, E. y Rodríguez Sehk, P. (1997). Más allá del dilema de los métodos. La investigación en ciencias sociales. Bogotá: Grupo Editorial Norma.

Bruner, Jerome. (2000). La educación, puerta de la cultura. Visor, Col. Aprendizaje, Madrid. España. (3 ed.) pág. 12

Cortina Orts, Adela. (2007). Ética de la razón cordial. Nobel, Oviedo.

Díez Jorge, María Elena y Sánchez Romero,
Margarita. (2010) Género y paz. Barcelona. España. Icaria.

Espinosa, Martín. (2018). Transformación social. Disponible en: https://www. excelsior.com.mx/opinion/martin-espinosa/ transformacion-social/1280914. Fecha de consulta 27 de noviembre de 2019.

Fisas Armengol, Vicent. (2011) Educar para una cultura de paz. España. (s. ed.)

(1998) Cultura de paz y gestión de conflictos. Barcelona, Icaria.

Galtung, Johan. (2003). Violencia cultural. Gernika-lumo.

García Vallinas, Eulogio. (2013) Hacia una forma más holística y dialógica para educar para la paz. Cultura de paz para la educación. Grupo Editorial Universitario.

Universidad de Nariño. (2014). Reglamento Practica pedagógica integral e investigativaPPII, Facultad de Educación. p. 10.

Fernández, Ana María. (1993) Las mujeres en la imaginación colectiva. Una historia de discriminación y violencias. Argentina. Paidos.

Flórez, Ochoa. (2005) Pedagogía del conocimiento. Bogotá: 2 edición. Ed. McGraw Hill.

Freire, Paulo. (1970). Pedagogía del Oprimido. New York: Herder \& Herder.

Gil Jaurena, Inés. (2013). Enfoque intercultural y animación sociocultural: convergencias y reflexiones. Revista Iberoamericana de Educación.

Luke, Carmen. (1999). Feminismos y pedagogías en la vida cotidiana. España. Morata. 
MagallónPortolés, Carmen. (1993). Hombres y mujeres: el sistema sexo-género y sus implicaciones para la paz. Mientras tanto, n54, pp. 61-76.

(2006). Mujeres en pie de paz. Madrid. Siglo $\mathrm{XXI}$.

Martínez, Miguel. (2006). La investigación cualitativa. Caracas: Ed. Trillas.

Martínez López, Cándida (1998). Las mujeres y la paz en la historia. Aportaciones desde el mundo antiguo. España. Instituto de la paz y los conflictos. Universidad de Granada.

Mayor Zaragoza. (2003). Educación para la paz. Educación XXI: Revista de la Facultad de Educación, № 6, 2003 (Ejemplar dedicado a: Educación para la paz). Madrid. págs. 17-24.

Monje Álvarez, Carlos Arturo. (2011). Metodología de la investigación cuantitativa y cualitativa. Guía didáctica. Universidad Sur Colombiana.

Muñoz, Francisco Adolfo. (2001). Historia de la paz. Tiempos, espacios y actores. Granada: Editorial Universidad de Granada. España

(2001). La paz imperfecta. Granada: Editorial Universidad de Granada. España.

(2004). Manual de paz y conflictos. Granada: Editorial Universidad de Granada. España.

et al. (2005). Investigación para la paz y los Derechos Humanos desde Andalucía.
Universidad de Granada. España.

Sambrano, Jazmín. (2007). Revista educación en valores. ISSN, 1690-7884. N8, España. págs. 52-55

Shiva, Vandana. (1995). Abrazar la vida: mujer, ecología y supervivencia, Madrid, Horas y Horas.

Shiva, Vandana, MIES, María. (1998). La praxis del ecofeminismo: biotecnología, consumo, reproducción. España. Icaria.

Thomas, Florence. (2006). Conversaciones con Violeta. Historia de una revolución inacabada. Bogotá. Colombia. Aguilar.

Valencia Parisaca, Narciso (1994). La Pachamama. Revelación del dios creador. Quito. Ecuador. Abya-Yala.

Freire, Paulo (2010). Pedagogía de la autonomía. México. Editorial Siglo XXI.

Freire, Paulo (2010). La educación en la ciudad. Editorial Siglo XXI. 


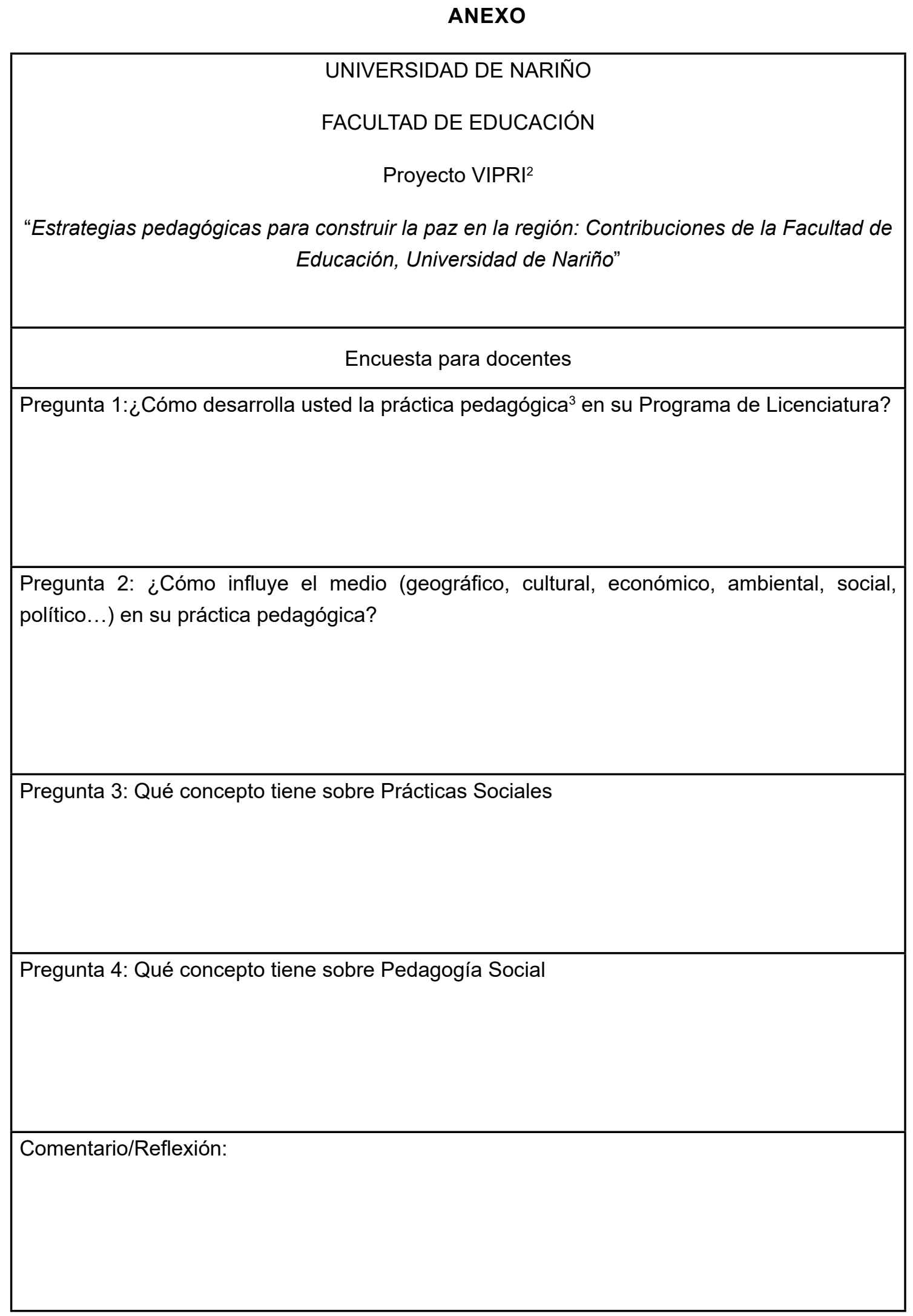




\section{(FOOTNOTES)}

$1 \quad$ La categoría correspondiente a interacción social es recurrente por las características de la temática analizada objeto de esta investigación y derivada de las experiencias descritas por los sujetos participantes.

2 Vicerrectoría de Investigaciones, Posgrados y Relaciones Internacionales de la Universidad de Nariño.

3 Práctica pedagógica: "se define como la construcción teórico-formal, que representa la realidad estudiada y se fundamenta científicamente en las teorías de la educación y la pedagogía; metodológicamente se basa en los diferentes enfoques de investigación y responde a una necesidad histórica concreta: la formación del maestro crítico, creativo, reflexivo, investigador y comprometido con los valores de la convivencia, el pluralismo, la solidaridad y la justicia social". Universidad de Nariño. (2014). Reglamento Practica pedagógica integral e investigativa-PPII, Facultad de Educación. p. 10. 